\title{
A Validation Study of a Kinect Based Body Imaging (KBI) Device System Based on ISO 20685:2010
}

\author{
Sara BRAGANÇA ${ }^{* 1}$, Miguel CARVALHO ${ }^{1}$, Bugao $\mathrm{XU}^{2}$, Pedro AREZES ${ }^{1}$, Susan ASHDOWN ${ }^{3}$ \\ ${ }^{1}$ University of Minho, Portugal; \\ ${ }^{2}$ University of Texas at Austin, USA; \\ ${ }^{3}$ Cornell University, USA \\ http://dx.doi.org/10.15221/14.372
}

\begin{abstract}
To replace the traditional anthropometric data collection processes with the 3D acquiring system it is important that the validity of the data is not compromised. To do this, a validation study, based on the guideline of ISO 20685, can be performed. This paper presents the results of a comparison between traditional measurements and measurements taken with a 3D acquiring system using only four Kinect sensors. The results obtained were then compared with the maximum allowable error indicated in ISO 20685 , concluding that this system cannot give sufficiently reliable data that can substitute the manual procedures.
\end{abstract}

Keywords: kinect, manual measurements, validation study

\section{Introduction}

With the diffusion of digital devices for body scanning, the science of anthropometry gained a new powerful tool that allows performing deeper investigations on human body shape. The new digital shape analysis tools allow to rethink the anthropometry science since, in the digital environment, the measurements are not limited to the traditional one dimensional ones, and instead it is possible to have complex geometrical features (i.e. curvatures and partial volumes). 3D body scanners have revolutionized anthropometric data acquisition, being more practical, accurate, fast and, when compared to traditional anthropometry, less expensive.

Currently, there are several types of imaging techniques to create full body images: laser line systems; structured light systems; multi-view camera systems; and millimeter waves [1].

It is possible to find in the market several three-dimensional body scanners that use different imaging techniques. The Microsoft Kinect is one of the newest available body scanners that uses a structured light system. Recent research and development has shown that the Kinect sensors can be used to create a system for human body imaging with similar accuracy to the expensive and complex 3D body scanning systems. When compared to many traditional systems, the $\mathrm{KBI}$ does not require large amounts of dedicated space or costly equipment. The KBI does not have moving parts, which reduces maintenance cost as well.

It is always important that body measurements obtained from 3D systems are sufficiently close to those produced by traditional methods so that they can be substituted for one another without compromising the validity of the data. To confirm the accuracy of the data gathered with the system that is being used, the procedures described in ISO 20685:2010 can be followed. The first step is to perform a validation study to verify if the $3 \mathrm{D}$ system gives sufficiently reliable results. This can be known if the $95 \%$ confidence interval for the mean of the difference, $d$, between the scan value and the measured value is within the limit values defined in ISO 20685:2010.

As such, the purpose of this paper is to perform a validation study for the system described using a sample of 40 test subjects (to ensure $95 \%$ confidence for large circumferences such as chest, waist, and hip, which are particularly difficult to measure for both traditional and 3D measurement systems). The validation test subjects will reflect approximately the same range of body sizes and shapes and an equal number of males and females will be surveyed.

\section{Literature Review}

Microsoft released, in November 2010, an inexpensive (approximately €100) accessory device known as Kinect. Kinect (Figure 1) is an input device that senses motion and was intended to be used as an alternative game controller for Xbox 360 video game consoles. Nevertheless, Microsoft Kinect can be used for numerous other applications besides games, a fact that brought the interests of the scientific and engineering communities. 


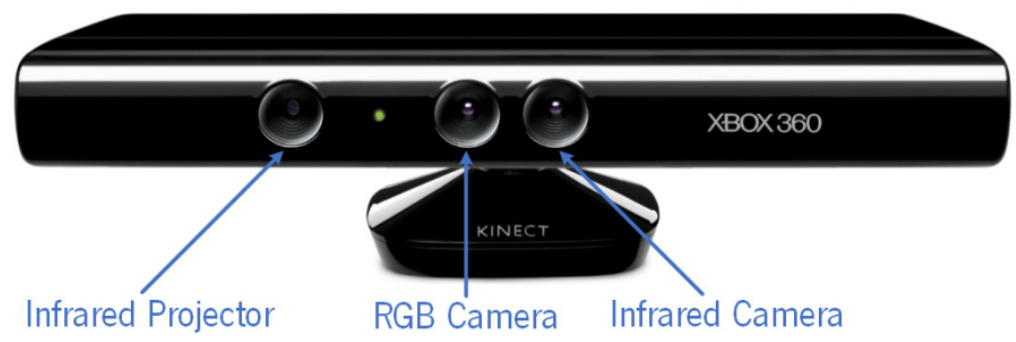

Fig. 1. Microsoft Kinect.

The Kinect sensor offers portable 3D motion capture capabilities that overcomes some limitations of the existing body scanning systems. Kinect allows Xbox 360 to detect the position of the players' body parts and follow the movements by identifying and tracking their joints.

The Microsoft Kinect sensor has its place in the class of devices known as depth cameras. It covers 3D space about 4 meters in depth and an angular field of view of 30 degrees to right and left. The key part of the Kinect's skeleton tracking is the depth sensor, that consists of an infrared laser projector and an infrared video camera mounted within the sensor bar. The system uses the infrared camera to detect a speckle pattern projected onto objects in the sensor's field of view.

The Kinect is able to create a $3 \mathrm{D}$ map of these objects by measuring deformations in the reference speckle pattern. Using this 3D map and a randomized decision forest of 3 trees, each trained by 300,000 images, Kinect is capable of specifying body joints' positions in 30 frames of still pictures per second, automatically determining anatomical landmarks on the body, such as joint centers, in close to real time [2]. The other components of Kinect are an RGB video camera, which provides color data for its depth map, an array of four microphones and a motorized base, which controls the tilt angle of the head.

The feature that distinguishes Microsoft Kinect from the other systems is the provision for skeletal and joint structure tracking to be captured by the camera. Its built-in ability to estimate joint center locations has made it a revolutionary device. Kinect can be seen as a 3D marker-less motion capture system because it provides a simplified skeleton (composed by 15 joints) in real time without the need for special clothes or other equipment.

Due to the simplification of the skeleton it cannot be used for extremely accurate studies. However, it is possible to use it when there is no need for such accuracy, like in clothes or shoes sizing, indirect fat measurement or clinical rehabilitation [3]. Recent research has shown that the Kinect system is capable of creating 3D human models with similar accuracy to the expensive and complex 3D body scanning systems [4]. When compared to many traditional systems, the Kinect does not require markers to be placed on participants before data collection, which reduces the data collection time, but also reduces the data accuracy, bringing many errors (e.g. pose estimation [5, 6]). According to [7] there are two sources of error that compromise the Kinect's accuracy:

- The error of the depth sensor in the position measurement of points;

- The error of the image processing software in determining the joints' positions from the depth sensor data.

To overcome this limitation, [5] presented a more accurate tracking algorithm for the Kinect. However, this increase in performance comes at the cost of execution speed. With the promise of rapidly collecting anthropometric data, many researchers tried to obtain data directly from 3D images, often known as point clouds. Early studies used high-quality point clouds generated by expensive equipment in ideal environments and used machine learning and image processing techniques to estimate anthropometric features [8, 9]. The CAESAR (Civilian American and European Surface Anthropometry Resource Project) data are widely used since they provide both high-fidelity point clouds sampled under highly controlled environments and corresponding anthropometric data obtained by physical measurement. However, the more recent work of [10] presents the possibility to extract anthropometry with this low-cost sensor and with participants dressed in regular clothing.

Testing the validity of the data obtained with three-dimensional body scanners is a subject that should be of concern in every study using this type of equipment. The work of [11] compared scan-derived measurements (obtained using a Vitronic Vitus-3D 1600 scanning system) with manual measurements in terms of accuracy and precision. The results obtained failed to meet the ISO 20685 criteria but were accurate enough to be used for apparel applications. [12] also tried to identify the differences between manual measurements and measurement obtained with a Cyberware WB4 scanning system. As happened in the previous example, in this study the mean absolute difference of all measurements is larger than the maximum allowable error specified by ISO 20685. Nevertheless, they propose some guidelines for the effective use of scan measurements. 


\section{Methods}

\subsection{Apparatus}

Kinect systems can have different configurations according to the desired application. In this paper a new body image acquisition system that uses only four Kinect devices to capture the entire human body (as shown in Figure 2) will be presented.

This system was developed by University of Texas at Austin (USA). After the precise registration, the front and back views of the body can be merged and reconstructed to form a smooth and complete digital model that is scalable and rotatable on the computer screen. The digital model can be used for measurement extractions, custom clothing and virtual try-on. The KBI system provides both hardware and software for 3D body imaging, which is quick (< 4s imaging time), portable (on stands), compact $(2 \mathrm{~m} \times 1.5 \mathrm{~m}$ footprint), accurate $(\sim 1 \mathrm{~mm})$ and relatively economical. It has a viewing volume of $2 \mathrm{~m} \times$ $1 \mathrm{~m} \times 0.8 \mathrm{~m}$ (height $\mathrm{x}$ width $\mathrm{x}$ depth). The body measurements include many circumference, length and volume data at pre-defined landmarks.

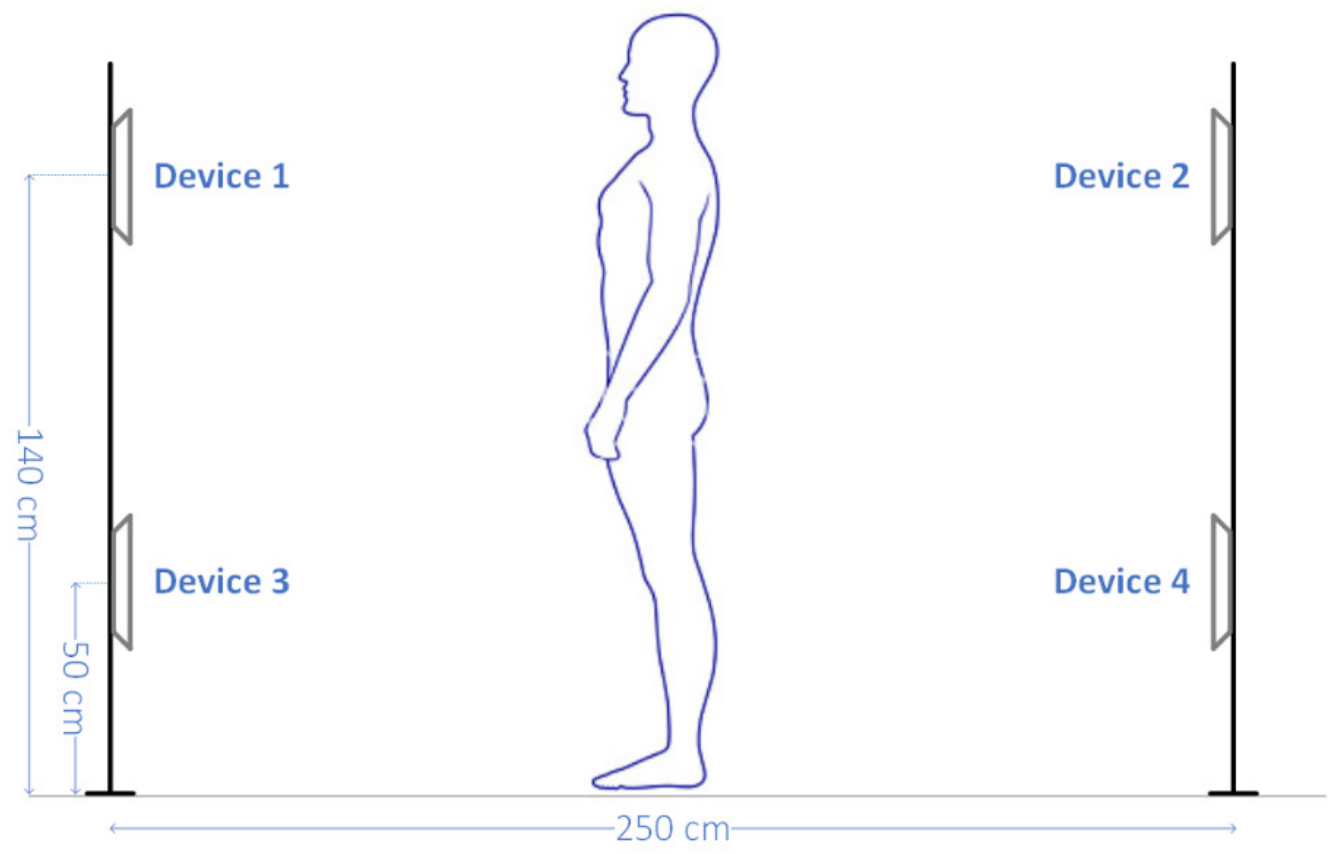

Fig. 2. Acquisition system.

\subsection{Subjects}

Thirty four subjects, fourteen females and twenty males, aged from 18 to 30 years old ( $M=24.09$; $\mathrm{SD}=2.84$ ) participated in this study. The selection of this age range was based on the assumption that there would be more similarities regarding average body shapes, history of medical problems and other age enforced conditions. This allowed for the results to be more feasible.

The fourteen females had an average age of 24 years old, an average height of $1.66 \mathrm{~m}$ and an average weight of $58 \mathrm{~kg}$. The twenty males had an average age of 24 years old, an average height of $1.77 \mathrm{~m}$ and an average weight of $75 \mathrm{~kg}$.

During the measurement procedures the subjects were wearing their own underwear or in some cases swimwear. To be noted that the selected garments did not affect the shape of the body parts to be measured.

\subsection{Procedure}

It was asked for test subjects to stand in an upright position with legs and arms slightly apart from the body (as shown in Figure 3), so that the KBI system could capture the entire body. This postures corresponds to the standing position A described in ISO 20685 - the head is in the Frankfurt plan; the long axes of the feet should be parallel to one another and $200 \mathrm{~mm}$ apart; the upper arms are abducted to form a $20^{\circ}$ angle with the sides of the torso, and the elbows are straight; the palms face backward, and the subject is breathing quietly. 
As analyzing and comparing all human body part would be very challenging, it was decided to select only ten body parts to perform this study. The dimensions selected are the following:
1. Neck circumference
2. Waist circumference
3. Hip circumference
4. Mid-thigh circumference
5. Knee circumference
6. Calf circumference
7. Ankle circumference
8. Shoulder width
9. Across chest length
10. Across back length

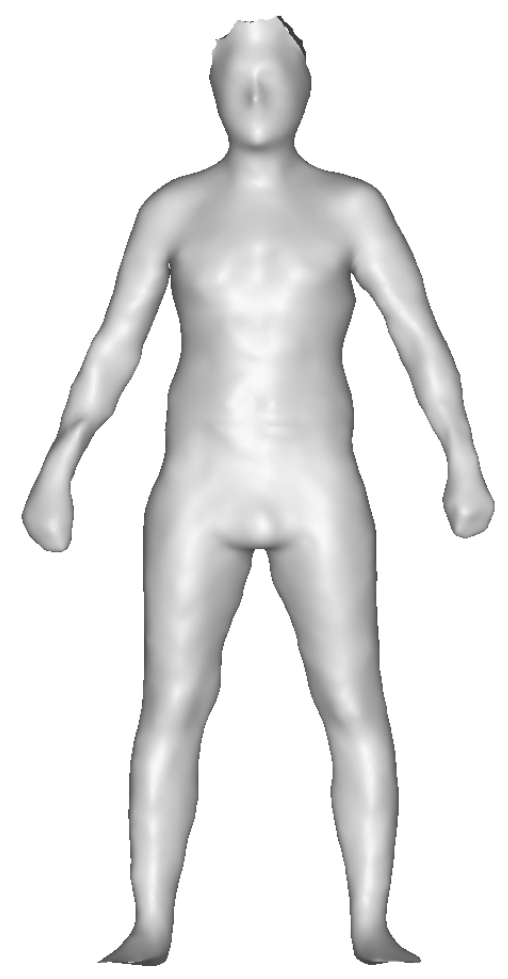

Fig. 3. Example of a subjects' scan.

Each subject was then measured five times, both manually (using a measuring tape) and automatically (using the Kinect). The subjects were first measured all five times automatically and then all five times manually (the ten body part always in the same sequence).

To avoid errors in the repetition of the measurements taken manually, a green marker was used to identify the necessary landmarks in all subjects.

After the collection of all necessary data it was necessary to calculate:

- the difference, $d$, between the scan value and the measured value ( $d=$ scan minus measure) for each variable and test subject;

- the mean of these differences for each variable;

- the associated standard deviation;

- the $95 \%$ confidence interval for the mean of scan-minus-measure differences.

\section{Results and Discussion}

To understand if the 3D system can give results sufficiently comparable to ISO 7250 methods (manual methods), and therefore that may be used in standards relying on ISO 7250 protocols, it is important to ascertain if the $95 \%$ confidence interval for the mean of can-minus-measure differences is within the plus or minus interval defined by the values in Table 4 of ISO 20685.

After measuring the thirty four test subjects the results obtained are the ones presented in Table 1. 
Table 1. Results of the comparison of manual and scanned dimensions (unit: $\mathrm{cm}$ ).

\begin{tabular}{|c|c|c|c|c|c|}
\hline \multirow{2}{*}{ Dimension } & \multirow{2}{*}{ Mean } & \multirow{2}{*}{$\begin{array}{l}\text { Standard } \\
\text { Deviation }\end{array}$} & \multicolumn{2}{|c|}{ 95\% Confidence Interval } & \multirow{2}{*}{$\begin{array}{l}\text { Allowable } \\
\text { error ISO } \\
20685\end{array}$} \\
\hline & & & Lower & Upper & \\
\hline Neck circumference & 0.24 & 1.72 & -0.35 & 0.84 & 0.4 \\
\hline Waist circumference & 2.14 & 2.69 & 1.21 & 3.08 & 0.9 \\
\hline Hip circumference & 5.63 & 338 & 4.45 & 6.81 & 0.9 \\
\hline Mid-thigh circumference & -2.38 & 2.63 & -3.30 & -1.46 & 0.4 \\
\hline Knee circumference & -2.56 & 1.64 & -3.13 & -1.98 & 0.4 \\
\hline Calf circumference & 0.15 & 1.51 & -0.37 & 0.68 & 0.4 \\
\hline Ankle circumference & 2.28 & 2.00 & 1.58 & 2.98 & 0.4 \\
\hline Shoulder width & -2.87 & 3.61 & -4.13 & -1.60 & 0.4 \\
\hline Across chest length & -0.22 & 2.51 & -1.10 & 0.65 & 0.5 \\
\hline Across back length & -1.14 & 3.10 & -2.23 & -0.06 & 0.4 \\
\hline
\end{tabular}

As can be seen in Table 1, there are some differences in the values obtained. Comparing the results of the $95 \%$ confidence interval with the maximum allowable error between extracted values and traditionally measured values given by ISO 20685 , it is possible to understand that, for almost every case the results are higher than what is recommended by the standard. The only two exceptions are the lower intervals of the neck circumference and the calf circumference. This means that this 3D system, as is currently, needs some improvements and is not as feasible as it could be to create anthropometric databases as accurate as those constituted by data from manual traditional methods. Despite this fact, these results can be used in other applications that require a lower level of accuracy, such as the garments industry.

Some reasons that justify these results are the fact that the subjects did not adopt the exact same posture during the five repetitions or that the measurements were not always done in the exact same place. Even with the existence of landmarks the placement of the measuring tape is hardly the same. Additionally, the automatic identification of landmarks in the software is subject to some error. Trying to identify the exact location of a landmark on the subject and on the software is virtually impossible, as the software does not recreate the images in color so that the location of the landmarks is impossible to see.

\section{Conclusions}

For every new 3D acquiring system destined to anthropometric uses, it is fundamental to ascertain its validity, understanding if it can be used to replace the measurements taken with traditional methods. In this paper it is presented a Kinect based body imaging technique (that uses four Kinect sensors) recently developed. The data of 34 test subjects was obtained and then the mean, the standard deviation and the $95 \%$ confidence interval were calculated for the mean of scan-minus-measure differences. These last results were then compared with the maximum allowable error defined in ISO 20685. This comparison made it possible to conclude that in this current state this acquisition system is not able to give results sufficiently comparable to the traditional methods defined in ISO 7250 . However, the accuracy of the scan-derived measurements is sufficient for other uses, such as apparel applications.

To overcome this situation, it is intended to enlarge the sample size and to readjust the system, recalibrating the several internal parameters. Action will also be taken to prevent subjects from moving during the scanning process. Additionally, it will be developed a system where the identification of the body landmarks on the software is possible. 


\section{References}

[1] H. Daanen and F. Haar, "3D whole body scanners revisited", in Displays, Vol. 34, No. 4, 2013, pp.270-275, http://dx.doi.org/10.1016/j.displa.2013.08.011.

[2] J. Shotton et al., "Real-time human pose recognition in parts from single depth images", in Proc of the IEEE Computer Society Conference on Computer Vision and Pattern Recognition, 2011, pp. 1297-304, http://dx.doi.org/10.1145/2398356.2398381.

[3] A. Baena et al., "Biomechanical validation of upper-body and lower-body joint movements of kinect motion capture data for rehabilitation treatments", in Proc of 4th International Conference on Intelligent Networking and Collaborative Systems (INCoS), 2012, pp. 656-661, http://dx.doi.org/10.1109/iNCoS.2012.66.

[4] A. Weiss et al., "Home 3D body scans from noisy image and range data", in Proc of IEEE International Conference on Computer Vision (ICCV), Barcelona, Spain, 2011, pp. 1951-1958.

[5] M. Ye et al., "Accurate 3d pose estimation from a single depth image", in Proc of IEEE International Conference on Computer Vision (ICCV), 2011, pp. 731- 738.

[6] R. Clark et al., "Validity of the microsoft kinect for assessment of postural control", in Gait \& Posture, Vol. 36, 2012, pp. 372-377, http://dx.doi.org/10.1016/j.gaitpost.2012.03.033.

[7] A. Mobini, "Accuracy of Kinect's skeleton tracking for upper body rehabilitation applications", in Disability and Rehabilitation: Assistive Technology, 2013, pp. 1-9, http://dx.doi.org/10.3109/17483107.2013.805825.

[8] Z. Azouz et al., "Automatic locating of anthropometric landmarks on 3d human models", in, 2006

[9] M. Maier, "Estimating anthropometric marker locations from 3-d ladar point clouds", Technical report, DTIC Document, 2011.

[10]M. Robinson and M. Parkinson, "Estimating Anthropometry with Microsoft Kinect", 2013

[11]J. Lu and M. Wang, "The evaluation of scan-derived anthropometric measurements" in Instrumentation and Measurement, Vol. 59, No. 8, 2010, pp. 2048-2054, http://dx.doi.org/10.1109/TIM.2009.2031847.

[12] H. Han et al., (2010). "Comparative analysis of 3D body scan measurements and manual measurements of size Korea adult females", in International Journal of Industrial Ergonomics, Vol. 40, No. 5, 2010, pp. 530-540, http://dx.doi.org/10.1016/j.ergon.2010.06.002.

[13]ISO 20685, "3D Scanning Methodologies for Internationally Compatible Anthropometric Databases", in International Organization for Standardization. Reference no. 20685-2005. ISO, Switzerland, 2005.

[14] ISO 7250, "Basic human body measurements for technological design", in International Organization for Standardization. Reference no. 7250-1996. ISO, Switzerland, 1996. 